\title{
Virtual Simulation of Seismic Forward Data Processing Based on LabVIEW
}

\author{
Huimei Yu, Xiang'e Sun \\ Electronics \& Information College of Yangtze University, Jingzhou, China \\ Email:yhm1990@sohu.com
}

How to cite this paper: Yu, H.M. and Sun, X.E. (2017) Virtual Simulation of Seismic forward Data Processing Based on Labview. Open Journal of Yangtze Gas and Oil, 2, $1-9$.

https://doi.org/10.4236/ojogas.2017.21001

Received: December 18, 2016

Accepted: January 16, 2017

Published: January 19, 2017

Copyright $\odot 2017$ by authors and Scientific Research Publishing Inc. This work is licensed under the Creative Commons Attribution International License (CC BY 4.0).

http://creativecommons.org/licenses/by/4.0/

\begin{abstract}
For those who have access to seismic exploration principles and seismic data processing initially, there are essential difficulties. The difficulties include the great amount of seismic data, complicated processing procedure, long processing period as well as costly software and facilities for data processing. It is an important issue for how to make the beginners understand and master the fundamental procedures of seismic data processing easily, and how to make the processing procedures just look like to be done with microcomputer. This article is emphasized on virtual simulating seismic forward data processing based on LabVIEW platform and LabSQL. LabSQL is LabVIEW's database kit, which is supplied by the $3^{\text {rd }}$ party for accessing the database. It is beneficial for beginners to control processing procedure efficiently. This method is worth being promoted.
\end{abstract}

\section{Keywords}

LabVIEW, Database, LabSQL, Seismic Forward Data Processing

\section{Introduction}

Seismic data processing refers to using the computer for processing seismic data, which is based on some principles of seismic. These principles are seismic waves' propagation theory, seismic exploration, a variety of methods of signal analysis and processing, etc. In this phase, seismic cross-sections, seismic velocity data and information can be generated. The information includes the characteristics of high signal-to-noise ratio, high resolution, high fidelity, and accurate imaging [1]. At present, softwares such as Promax, Focus, and Vista are used to process seismic forward data. In general, computer of high configuration is required. In order to let users understand data processing intuitively, the most effective method is simulating the processing, then creating the interface for users to see the details of processing. LabVIEW, developed by NI companies, is a kind of 
software based on the virtual instrument development platform of graphical programming. The software establishes hardware platform based on general computer, and implements test function by test software [2]. The software provides many control, their appearances are similar to the traditional instruments. It's beneficial to create the interface. The characteristics of graphical programming are simple and intuitive, so that writing the program is similar to the method of constructing the flow [3]. The software uses the data flow model to realize automatic multithreading, and because of the full use of multiprocessor, the program would run faster [4]. The article chooses LabVIEW and MYSQL database to develop a system to describe seismic forward data processing. There is no need to build complex work station or install seismic data processing software. It is convenient for users to learn process seismic forward data only by connecting to the Internet through a browser.

\section{Construct Platform for Data Processing}

In seismic data processing, observation system files will be loaded firstly. These files include shot points information, detection points information and observation system parameters. They are deposited in database as data form. Velocity spectrum and geological section, generated in processing, because of the great amount of data, they ought to be deposited as image form. Then images and data in the platform can be viewed. The platform is built by MYSQL database and LabVIEW. Database may be used for storing data and images of seismic. The function of LabVIEW is to design interface and call images with data mentioned above. In general, LabVIEW can be used to call the corresponding images and parameters to display when users select one step of processing.

Platform construction is the process of selecting an optimum method for LabVIEW to access the database. The next steps are establishing a connection and determining the seismic forward data processing stage. The data and images will be generated and stored automatically in the database. The designed interface in LabVIEW will allow users to read and represent corresponding data and images (Figure 1).

\section{Choose a Way to Link LabVIEW to MYSQL Database}

MYSQL database is needed to store the graphic data for data processing system. However LabVIEW does not have access to the database directly, which needs other auxiliary tools to realize the function. At present, the main methods are the followings [5]: 1) Using LabVIEW SQL Toolkit to access the database. The advantages are simple to operate and easy to understand But it is not free. 2)

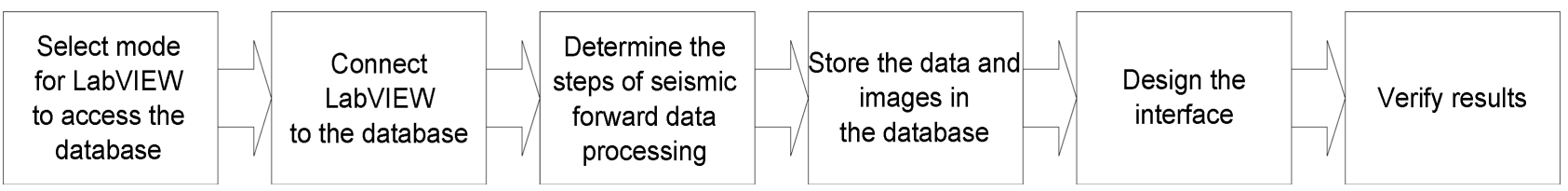

Figure 1. Establish the platform of seismic forward data processing. 
Using LabVIEW ActiveX function to call Microsoft ADO object, then accessing the database through SQL language. This method requires users to insight into SQL language. 3) Calling DLL (Dynamic Link Library) to access the database. Other language such as $\mathrm{C}++$ can be used to write the DLL. The disadvantage is that a complex programming should be done from the ground. 4) Using intermediate files to access data, such as disk file. This method cannot respond instantly, so it is not convenient to use. 5) Using LabSQL, which is a free kit developed by the $3^{\text {rd }}$ party. LabSQL encapsulates the complex underlying operation into a series of Vis with the help of Microsoft ADO and SQL language. These Vis are so easy to call that the method is simple for us. In conclusion, the article chooses the last method to access the database.

\section{Access the Database by LabSQL}

LabSQL connects to the database through ODBC, a common interface between the database and application. The application communicates with the database by accessing the ODBC. Before assessing the database, creating a data source in the ODBC data source manager is vital. It means assigning data source name and driver in ODBC is necessary. The specific steps are as follows [6]:

1) A database named "geology" should be created in MYSQL database.

2) "Management tools" and "data source (ODBC)" in Windows control panel can be chosen. Next step is to choose the add button in "user DNS" TAB of "ODBC data source manager".

3) It is essential to click add button in TAB of system DSN. When the dialog of data source Driver prompts, corresponding data source Driver should be chose. Here we choose MYSQL ODBC 5.3 ANSI Driver.

4) The steps are choosing database "geology" in database catalogue and filling "data source name" with "geology".

5) A new database named "geology" will be created in "ODBC data source manager".

\section{Design the Seismic Forward Data Processing}

Seismic forward processing refers to simplifying the actual complex earth medium and laws of seismic waves propagating. The characteristics can be researched when seismic waves spread in a simplified model by using the method of math or physics. Owing to the differences in lithology of subsurface rock stratum, the lithology information may be converted into the information of seismic waves. Then seismic data, collected in seismic waves, will be processed for theoretical research of wave field. The achievement is beneficial to guide the field acquisition of seismic exploration, processing and interpretation, etc.

Conventional seismic forward data processing (Figure 2) is designed to ensure that the final results reflect the characteristic of subsurface interface and tectonics. In order to determine the relative position of shot points and receive points, the observation system may be set up. After loading the data, for eliminating the interference of multiple waves and surface waves, the seismic traces 


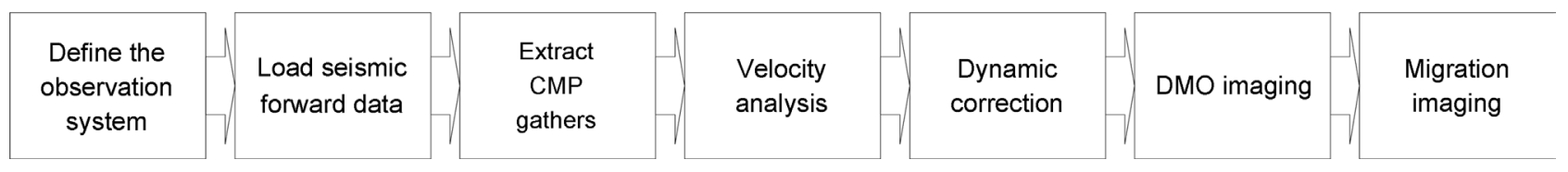

Figure 2. Seismic forward data processing in this article.

are arranged together. They come from the same center. It is called extracting common middle point (CMP) gathers. Velocity analysis is to get stack velocity spectrum, and prepare for the preliminary dynamic correction. After that the aim of suppressing or weakening interference waves can be achieved. For the sake of accurate result, three times of dip move out (DMO) velocity analysis are necessary to eliminate the influence of stratigraphic dip move out. The results will be processed by DMO imaging and migration imaging to realize pre-stack migration and improve the quality of section stacking. The function of the system is to describe the data processing in the form of images, which let users have a deeper understanding of the data processing.

\section{Design the MYSQL Database}

In seismic exploration, loading system is crucial because it will affect the quality of data processing. There is a perspective to introduce how the parameters and images of observation system stored in the database. The perspective is defining the observation system.

Observation system refers to the relative position of shot points and receiver points. In general, the wide azimuth observation system is defined that the ratio of transverse-to-longitudinal is more than 0.5 , otherwise it is narrow azimuth observation system. To explore the difference between the results on the basis of the two sets of observation systems, these systems ought to be stored in the database. Because of the difference of the observation directions, there are some contrasts in imaging velocity field. In addition to the two sets of systems, three observation systems of narrow azimuth with different directions are also needed.

Firstly select the database named "geology" in database directory. After that create a new data table and denominate it as "observation systems". Create fields that are related to observation systems. The fields include parameters of observation system. For example, the number of shots, the number of receive channels, the total number of receive channels, the distance of channel, the folds, and the ratio of transverse-to-longitudinal. It is essential to create field "path" to store the images paths of different observation directions. At last, numbering the images to make the front panel will make them convenient.

\section{Design the Interface}

The front panel should be easy to use and have multiple functions after entering the system. A main panel to put buttons should be created. Each button corresponds with one step of the data processing. These front panels also need to be created, everyone includes functions of showing images, jumping to the next step and terminating the whole system (Figure 3). 
At present all the images of data processing are stored in the database. The key of system design is to call and read corresponding images from the database by operating the front panel (Figure 4).

The function of the four ADO objects is to access data source. These string functions on the objects can create SQL language. After operating the front panel, the functions query the database and return paths of images. The role of other functions is to draw pictures according to paths.

For example, to define the observation system, the steps of designing the user interface are as follows: The function of the front panel is selecting a set of observation system. The program will access to the database and feedback the result to the front panel. So the front panel may provide different choices of observation directions and attributed images. Then the parameters corresponding observation systems can be listed in the interface. To users, understanding how to set the observation systems will affect the positional relation of geologic body. It is important to plot the location images of them on the interface. After entering the interface, it is easy to view the position of shot points, folds offset, images of azimuth attributes, and the corresponding parameters. Selecting the observation systems that have been set is the only thing should be done.

In Figure 5 there are some images of the system. The steps of data processing are listed in the main interface (Figure 5(a)). Choosing every button means showing corresponding results of processing. For example, after choosing the button of load seismic data, the system pops up the corresponding interface and shows the single shot record (Figure 5(b)). The Figure 5(c) means the result of choosing the button of NMO, the Figure 5(d) could appear if we choose the button of DMO imaging.

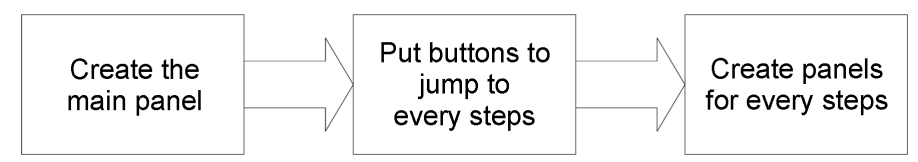

Figure 3. The flow chart of the front panel.

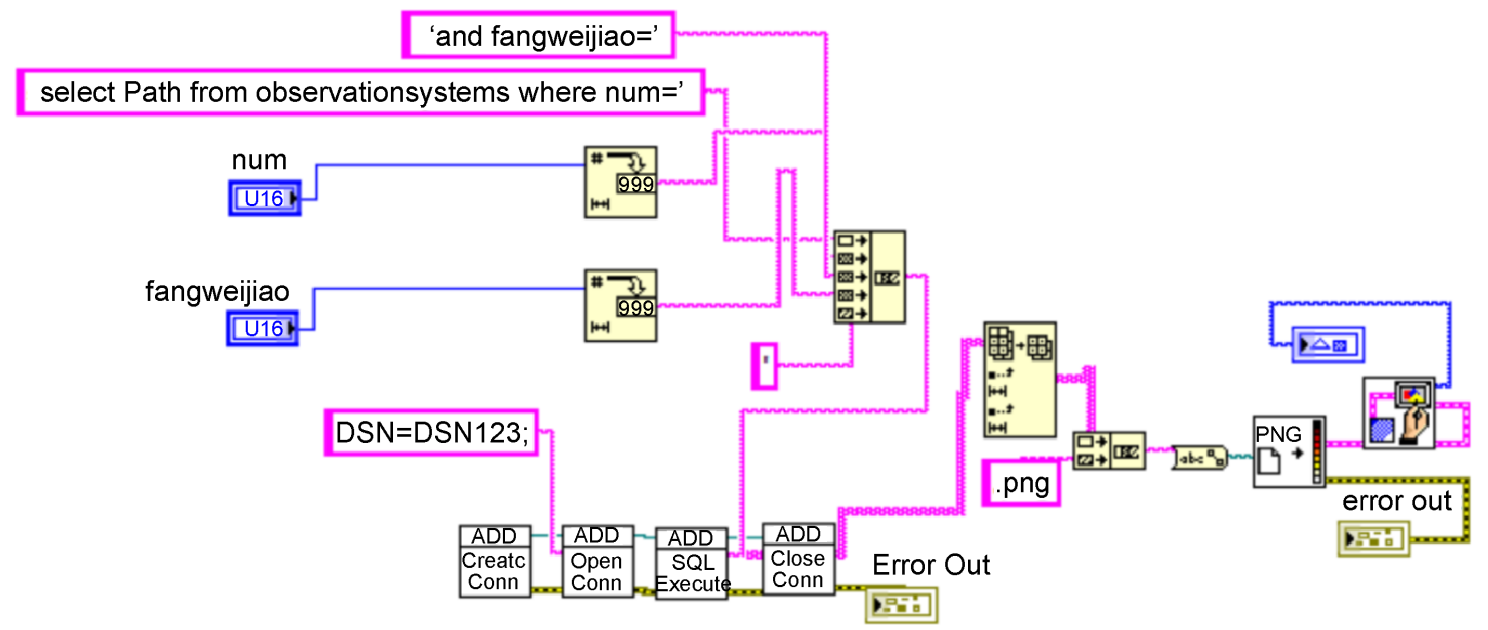

Figure 4. Call the images in the database. 


\section{Conclusions}

The article introduces using LabSQL supplied by the $3^{\text {rd }}$ party to access MYSQL database in the development environment of LabVIEW2012. The system realizes the function of calling data and images stored in the database by LabVIEW. Through seismic forward data processing, the results which are processed by the

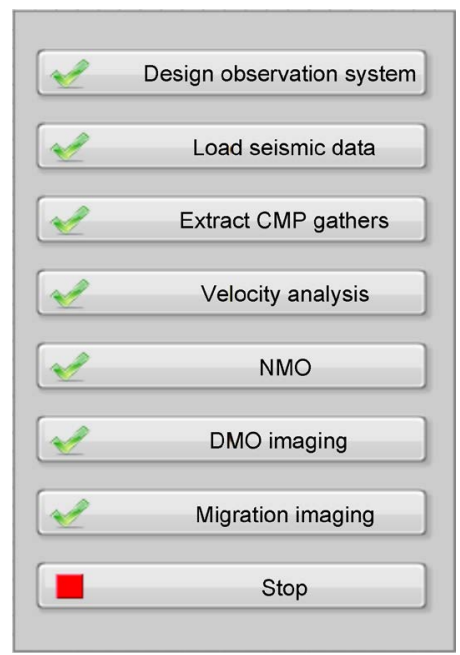

(a) The main interface of data processing

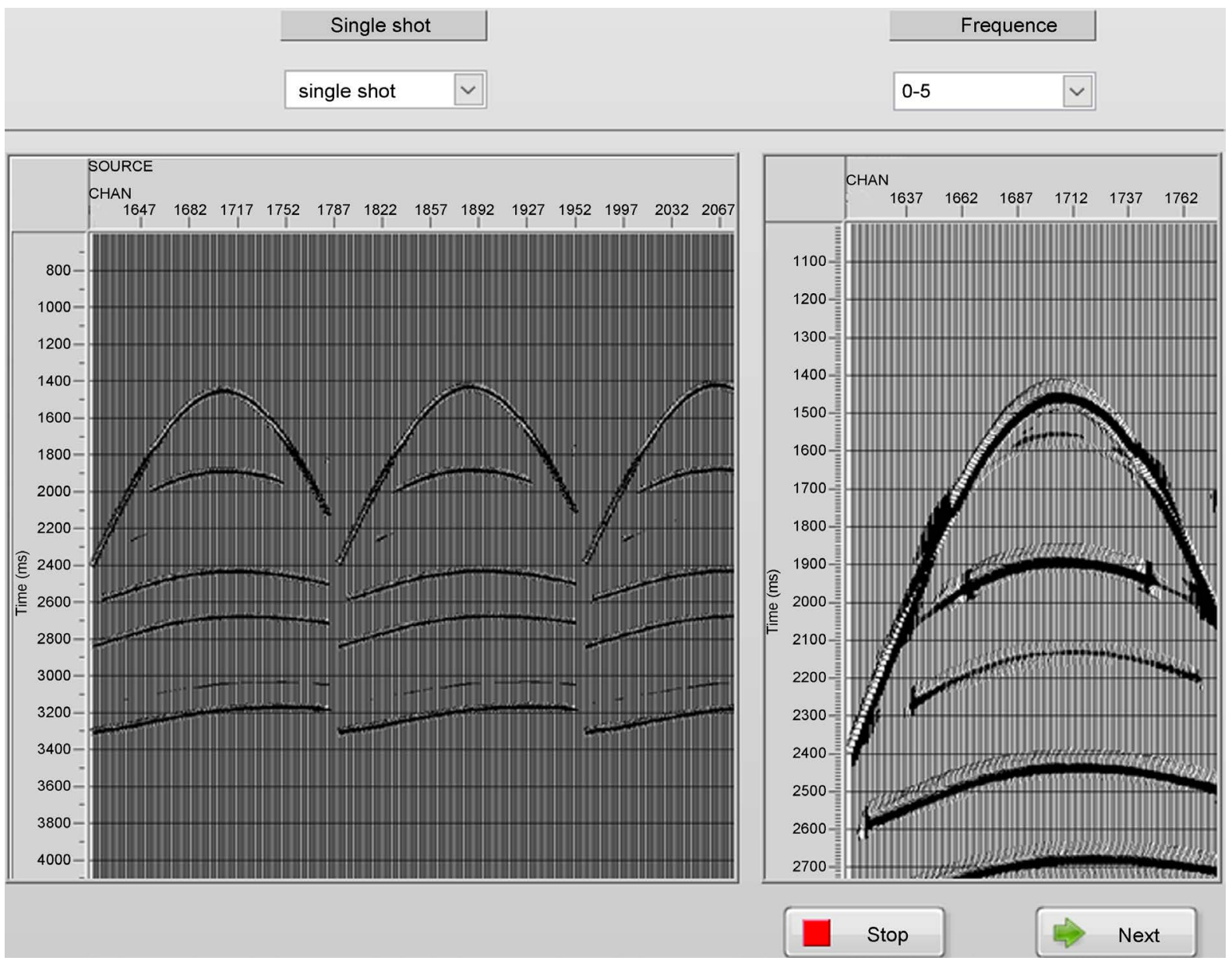

(b) Single shot record 


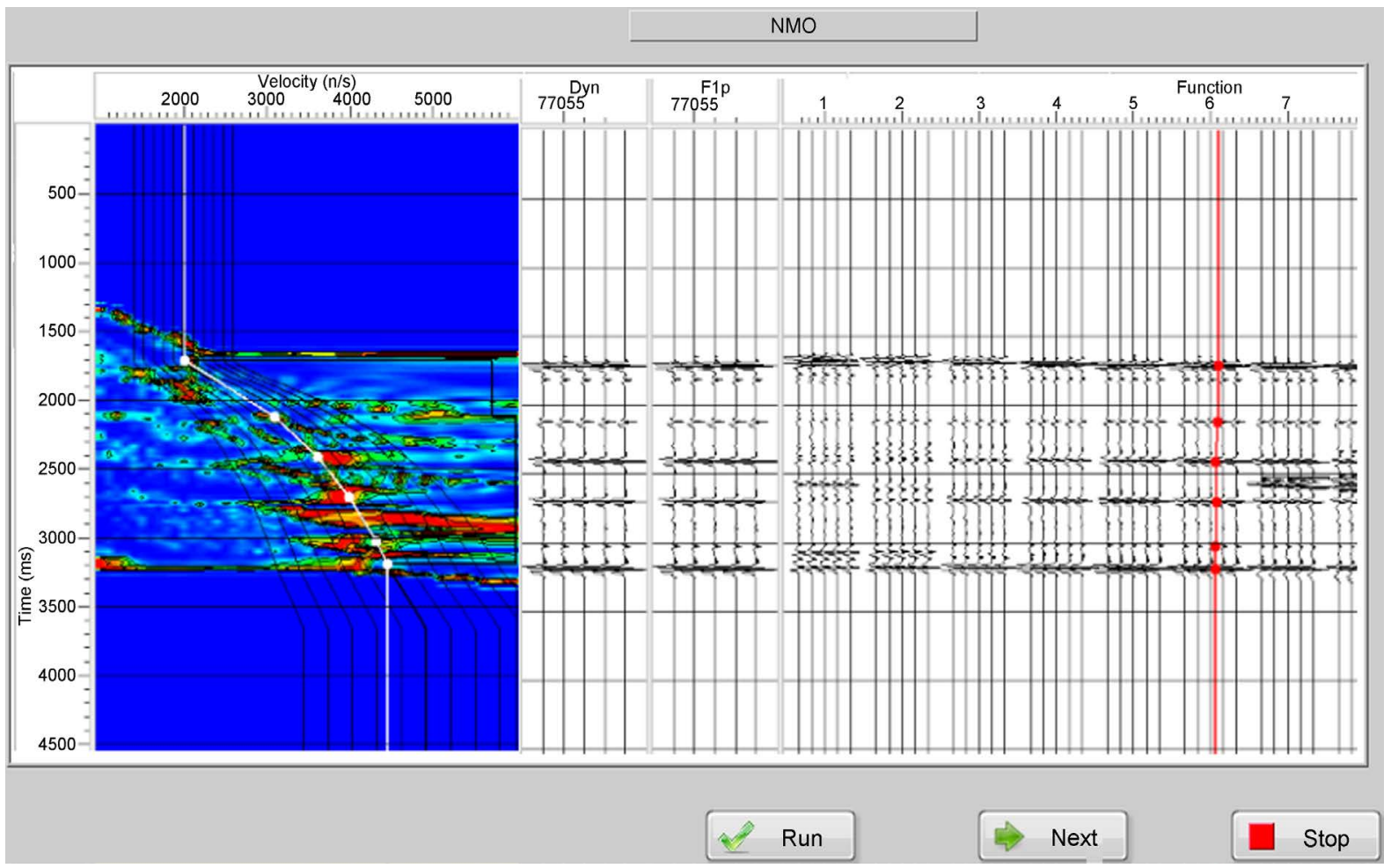

(c) Dynamic correction

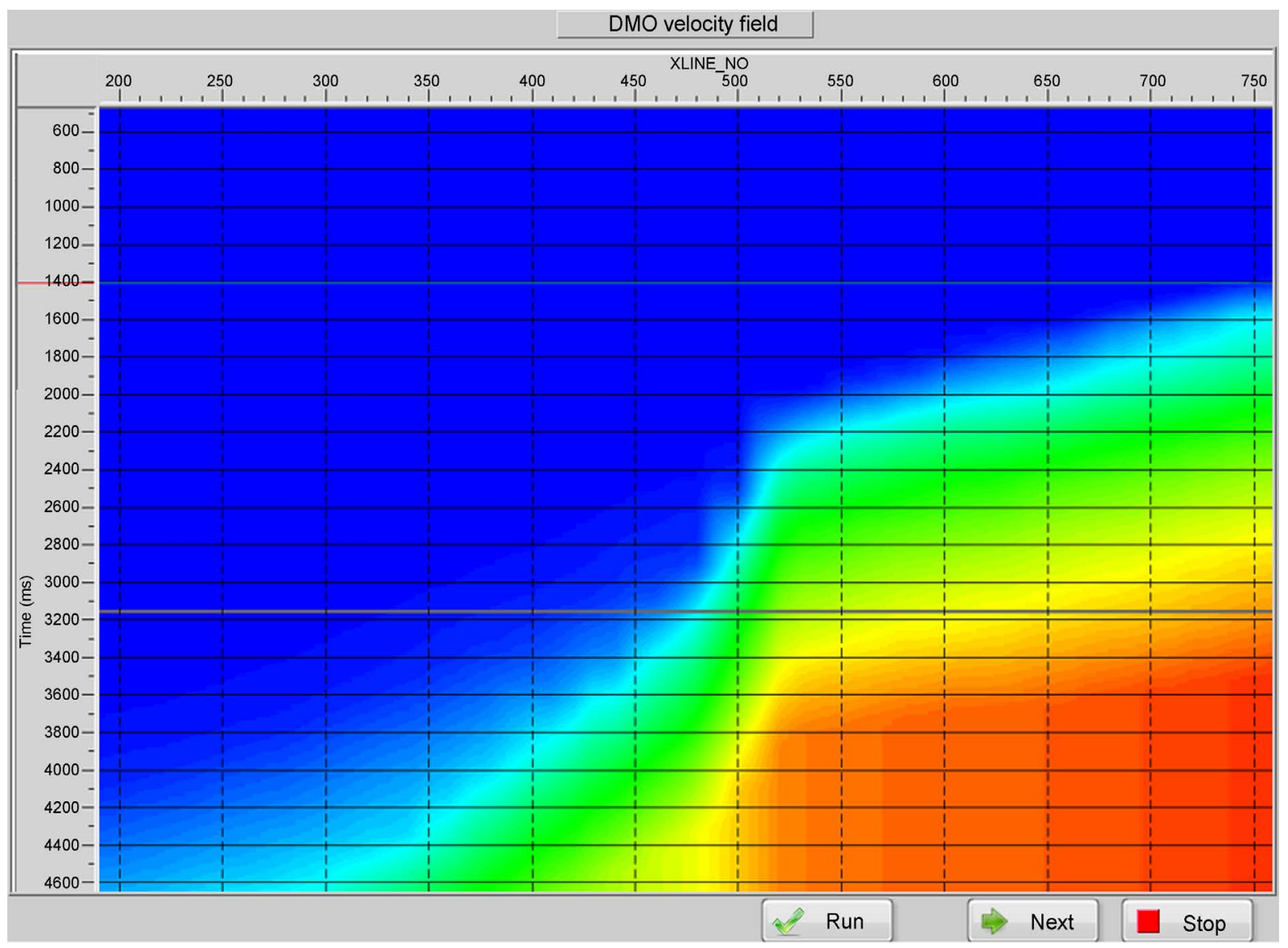

(d) DMO velocity field

Figure 5. Step charts of seismic forward data processing. 
processing methods based on the same observation system are different. For example, Figure 6 means comparing the results of DMO stack imaging and migration imaging based on the wide azimuth observation system. DMO stack imaging gets a consistent result in the stratum that changed slowly. To the contrary, migration imaging is suitable for the area with structure of severe changes. In addition, the system can reveal the results by the same method while the observation systems are different. We select the wide azimuth observation system and the narrow azimuth observation system as examples. The images of folds have obvious differences (Figure 7).

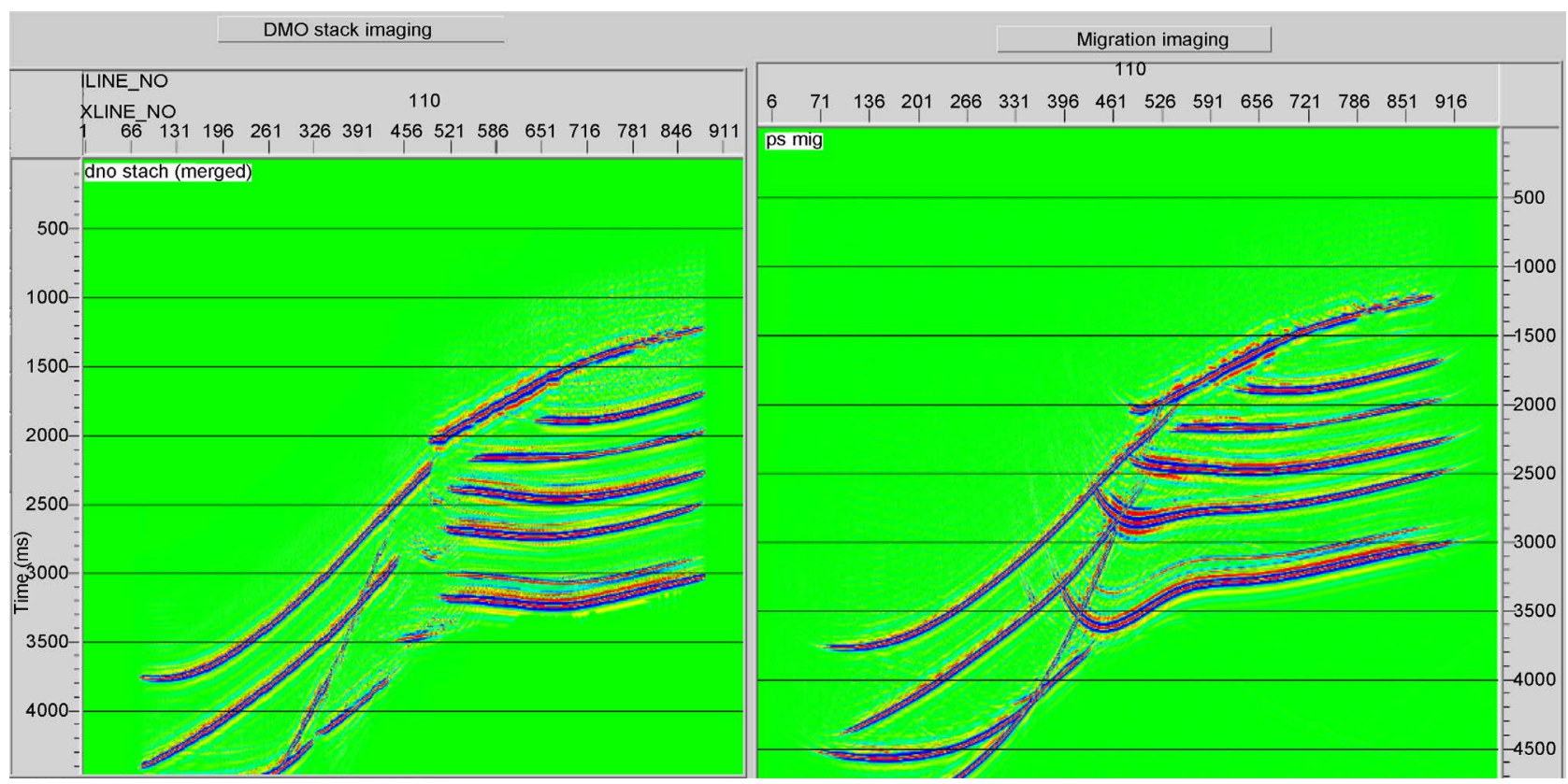

Figure 6. Migration imaging and DMO stack imaging.

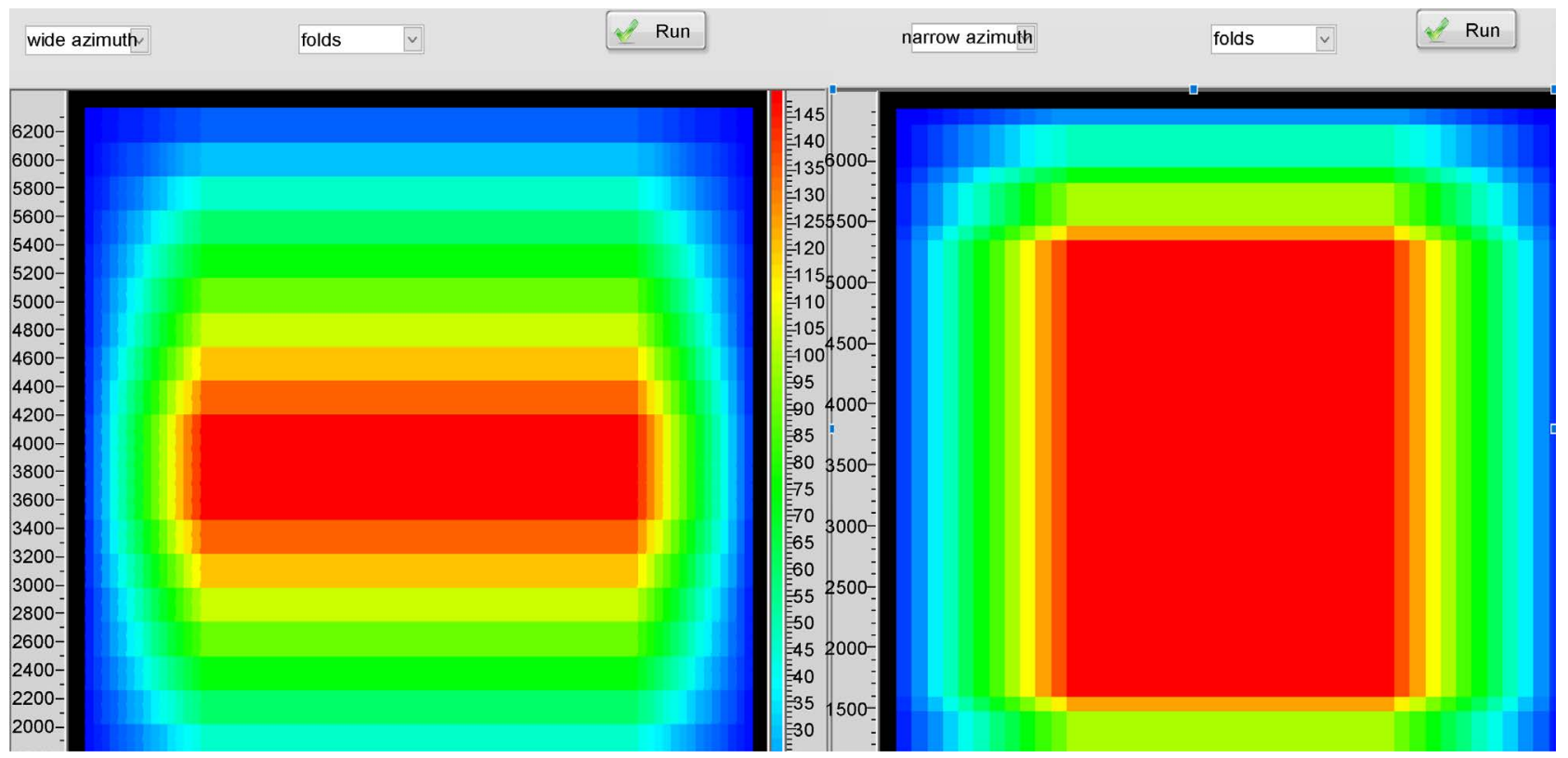

Figure 7. Folds of different systems. 
The idea can be proved to be feasible. The graphical feature of LabVIEW makes this system has a friendly interface, and easy to use [7]. It is meaningful for users to learn and understand seismic forward data processing without complex operation, with the help of this system.

\section{Funding}

Supported by Natural Science Foundation of China (Grant No: 51504039).

\section{References}

[1] Lu, J.M. and Wang, Y.G. (2009) The Principle of Seismic Exploration. 3rd Edition, University of Petroleum, Shandong.

[2] Liu, J.H. and Jia, H.Q. (2001) The Graphical Programming Language of Virtual Instrument, LabVIEW Course. Xidian University, Xi'an.

[3] Dong, W.B. and Wang, Q.J. (2011) APD Noise Equivalent Power Measurement System Based on Virtual Instrument Technology. Chinese Journal of Scientific Instrument, 32, 2635-2640.

[4] Chen, X.H. and Zhang, Y.H. (2007) Mastering LabVIEW 8.20 Program Design. Tsinghua University, Beijing.

[5] Bi, H. and Lv, F.C. (2006) Several Different Ways for LabVIEW to Access the Database. Microcomputer Information, 22, 131-134.

[6] Tang, Y.P. and Hou, Y.B. (2011) The Development of the Practice Teaching Platform and Access Database Based on LabVIEW. Computer Technology and Development, 21, 220-221.

[7] Fei, L. and Wang, B. (2012) Data Acquisition and Test System Design Based on LabVIEW. Journal of Chongqing University of Technology, 26, 38-41.

Submit or recommend next manuscript to SCIRP and we will provide best service for you:

Accepting pre-submission inquiries through Email, Facebook, LinkedIn, Twitter, etc. A wide selection of journals (inclusive of 9 subjects, more than 200 journals)

Providing 24-hour high-quality service

User-friendly online submission system

Fair and swift peer-review system

Efficient typesetting and proofreading procedure

Display of the result of downloads and visits, as well as the number of cited articles

Maximum dissemination of your research work

Submit your manuscript at: http://papersubmission.scirp.org/

Or contact ojogas@scirp.org 